\title{
A Case of Trigeminal Neuralgia Treated With Combination of Antihistamine, Montelukast, and Corticosteroid Nasal Spray
}

\author{
Asna Shahab ${ }^{1}$, Mydah S. Hashmi ${ }^{2}$, Zahoor Ahmed ${ }^{3}$, Agha S. Haider ${ }^{4,5}$, Aimen Haider ${ }^{6}$ \\ 1. Internal Medicine, Dow International Medical College, Dow University of Health Sciences, Karachi, PAK 2. Internal \\ Medicine, Army Medical College, Rawalpindi, PAK 3. Internal Medicine, King Edward Medical University, Mayo \\ Hospital, Lahore, PAK 4. Internal Medicine/Pulmonology, John Randolph Medical Center, Hopewell, USA 5. Internal \\ Medicine/Pulmonology, Southside Regional Medical Center, Hopewell, USA 6. Faculty of Health, York University, North \\ York, CAN
}

Corresponding author: Asna Shahab, asna_s93@yahoo.com

\begin{abstract}
Trigeminal neuralgia (TN) is a nerve disorder of the face associated with excruciating pain that occurs in paroxysms and can be initiated by even mild cutaneous stimuli. Diagnosis of TN is based on the patient's history and the diagnosis of exclusion. The first-line treatment usually comprises carbamazepine or oxcarbazepine. Herein we present a case of a 47-year-old female, diagnosed with idiopathic TN. Initially, she was commenced on carbamazepine, and later, she was switched to sodium valproate without any noticeable relief. However, she responded to treatment with combination therapy comprising antihistamine, montelukast, and corticosteroid nasal spray. To the best of our knowledge, no such case has been reported previously.
\end{abstract}

Categories: Neurology, Pain Management, Allergy/Immunology

Keywords: trigeminal neuralgia, antihistamine, montelukast, corticosteroid nasal spray, refractory pain, allergy

\section{Introduction}

Based on the available clinical data to date, the first-line treatment for trigeminal neuralgia (TN) is carbamazepine and oxcarbazepine. Other drugs such as phenytoin, baclofen, gabapentin, sodium valproate, lamotrigine, and clonazepam can be added if carbamazepine is unresponsive [1,2]. The pathophysiology of neuropathic pain is complex and associated with an increased level of excitatory neurotransmitters and neuropeptides such as histamine, bradykinin, 5-hydroxytryptamine, glutamate [3]. We present the case of a 47-year-old female with right-sided facial pain and mild numbness that readily responded to a combination of an antihistamine, montelukast, and corticosteroid nasal spray.

Review began 11/28/2020 Review ended 12/07/2020 Published 12/22/2020

() Copyright 2020

Shahab et al. This is an open access article distributed under the terms of the Creative Commons Attribution License CC-BY 4.0., which permits unrestricted use, distribution, and reproduction in any medium, provided the original author and source are credited.

\section{Case Presentation}

A 47-year-old Caucasian female presented with excruciating right-sided facial pain and mild numbness for the past one and a half years. The pain was episodic in nature, occurring every other day, lasting a couple of minutes. The attacks were triggered by the intake of cold drinks and spicy food. The attacks were not associated with any additional symptoms, particularly visual disturbance, headaches, shoulder and pelvic pain, and allergic conditions. Her past medical history was significant for hypothyroidism. On neurological exam, the patient was alert, awake, and oriented, no sensory or motor deficit was noted, and cranial nerves (CN) II-XII were grossly intact. The rest of the rheumatological, otolaryngology, dental, and allergy examination, MRI of the brain and sinuses, and blood and skin allergy workup were unremarkable. Blood work comprised of complete blood count, alanine transaminase, erythrocyte sedimentation rate, C-reactive protein, rheumatoid factor, complement (C3/C4), antinuclear antibody (ANA), double-stranded DNA (dsDNA), and thyroid-stimulating hormone; all were within the normal range (Table 1). 


\section{Cureus}

\begin{tabular}{|c|c|c|c|}
\hline Blood Work & Results & Reference Range & Unit \\
\hline Red blood cell count & 4.69 & $4.0-5.1$ & XE12/L \\
\hline White blood cell count & 7.7 & $4.0-11.0$ & x E9/L \\
\hline Platelets & 259 & $150-400$ & $x$ E9/L \\
\hline Eosinophils & 0.1 & $0.0-0.5$ & $x$ E9/L \\
\hline Neutrophils & 5.2 & $2.0-7.5$ & x E9/L \\
\hline Lymphocytes & 2.0 & $1.0-3.5$ & $\mathrm{X} E \mathrm{E} / \mathrm{L}$ \\
\hline Alanine aminotransferase & 17 & $<36$ & $\mathrm{U} / \mathrm{L}$ \\
\hline Erythrocyte sedimentation rate & 16 & $2-30$ & $\mathrm{~mm} / \mathrm{hr}$ \\
\hline C-reactive protein & 1.9 & $<5$ & $\mathrm{mg} / \mathrm{L}$ \\
\hline Rheumatoid factor & $<10$ & $<14$ & $10 / \mathrm{mL}$ \\
\hline Complement component 3 (c3) & 1.37 & $0.90-1.80$ & $g / L$ \\
\hline Complement component 4 (c4) & 0.37 & $0.15-0.53$ & $\mathrm{~g} / \mathrm{L}$ \\
\hline Albumin & 46 & 35-42 & $g / L$ \\
\hline Immunoglobulin class A (IgA) & 1.22 & $0.54-4.17$ & $g / L$ \\
\hline Immunoglobulin class M (IgM) & 1.32 & $0.30-2.30$ & $\mathrm{~g} / \mathrm{L}$ \\
\hline Immunoglobulin class G (IgG) & 9.62 & $6.00-16.00$ & $\mathrm{~g} / \mathrm{L}$ \\
\hline Immunoglobulin class E (IgE) & 2.0 & $<100$ & $\mathrm{kU} / \mathrm{L}$ \\
\hline Thyroid-stimulating hormone & 1.64 & $0.32-4.00$ & mlU/L \\
\hline Ferritin & 54 & $5-272$ & $\mathrm{ug} / \mathrm{L}$ \\
\hline Vitamin B12 & 234 & 138-652 & $\mathrm{pmol} / \mathrm{L}$ \\
\hline Antinuclear antibody (ANA) & $<1$ & Negative & Negative \\
\hline Anti-double-stranded DNA & $<1$ & $<5$ & IU/mL \\
\hline Tree mix & Negative & $<0.35$ & KU/L \\
\hline Dust mite & Negative & $<0.35$ & $\mathrm{KU} / \mathrm{L}$ \\
\hline Grass mix & Negative & $<0.35$ & $\mathrm{KU} / \mathrm{L}$ \\
\hline Mould mix & Negative & $<0.35$ & KU/L \\
\hline Chicken feathers & Negative & $<0.35$ & KU/L \\
\hline Cat and dog dander & Negative & $<0.35$ & KU/L \\
\hline Food allergy & Negative & $<0.35$ & $\mathrm{KU} / \mathrm{L}$ \\
\hline
\end{tabular}

TABLE 1: Comprehensive blood workup

Based on the history, examination, and laboratory findings, a clinical diagnosis of TN was made. The patient was initially started on carbamazepine $100 \mathrm{mg}$ two times a day with no relief; eventually, the dose was increased to $1200 \mathrm{mg}$ per day for three months with no improvement. Later, she was switched to sodium valproate $200 \mathrm{mg}$ per day for one month without any noticeable relief. After the allergy and clinical immunology consultation, though the skin and blood allergy workup were noncontributory, the patient was started on a combination therapy that consisted of tablet rupatadine $10 \mathrm{mg}$, tablet montelukast $10 \mathrm{mg}$, and Avamys nasal spray $27.5 \mathrm{mcg}$ two sprays per nostril once daily every day for three months initially. On follow-up, the patient reported significant pain relief, the frequency of the episodes reduced from every other day to 11-12 episodes per year. 


\section{Discussion}

$\mathrm{TN}$ is defined as paroxysmal attacks of severe shooting, stabbing pain in one or more divisions of the trigeminal nerve that lasts from a fraction of a second to two minutes. The pain is initiated by chewing, light pressure, or gentle stroke on the affected side [4]. The episodes of neuralgia may vary from patient to patient, ranging from nil to 50 per day [5]. In more than half of the patients, there is complete remission between the episodes ranging mostly for a few months [5].

$\mathrm{TN}$ can be classical or secondary. The classical TN is due to compression of the trigeminal nerve, mostly by an artery at the level of the nerve root [4]. The most commonly involved artery is the superior cerebellar artery [6]. The nerve root compression results in demyelination, atrophy, and displacement of the nerve. The secondary $\mathrm{TN}$ is due to a specific underlying disease such as herpes zoster infection, multiple sclerosis, space-occupying lesion, or tumor at a cerebellopontine angle [4].

TN is a clinical diagnosis. The International Classification of Headache Disorders (ICHD) in its third edition described that the diagnosis of TN requires to fulfill all of the following criteria. There are paroxysms of severe unilateral, non-radiating pain in one or more divisions of the trigeminal nerve stimulated by innocuous stimuli. The pain is severe in intensity and has stabbing, electric shock-like, or shooting characteristics. The duration of pain is variable but ranges from a fraction of a second to two minutes. The pain cannot be explained by any other medical illness in ICHD-3 diagnosis [4]. The incidence of TN increases with age, with a higher incidence in females than males [7].

The first-line treatment for both classical and secondary TN is oral carbamazepine and oxcarbazepine [8]. Add-on therapy with other anticonvulsants, including lamotrigine, pregabalin, gabapentin, and skeletal muscle relaxants such as baclofen, is prescribed to patients with inadequate control of symptoms or emergence of adverse effects at a high dose [8].

A case report of a 48-year-old female by Horton et al. in 1948 showed significant relief in symptoms of TN after administration of first-generation antihistamines. The patient had exacerbation and severe episodes of $\mathrm{TN}$ in the spring. The seasonal exacerbation and alleviation of TN symptoms after administration of antihistamine hinted at an underlying allergic etiology [9].

A follow-up clinical study was performed by Hanes on $183 \mathrm{TN}$ patients. Combination therapy of antihistamines with hydrochloric acid and histamine desensitization resulted in complete relief of symptoms in $57.3 \%$ of patients and partial relief of symptoms in $11.4 \%$ of patients [10]. Currently, a clinical trial on TN patients is being conducted by China Medical University Hospital to assess the therapeutic efficacy of a combination of antihistamine and acupuncture in relieving the neuralgia symptoms. More data is needed to validate the use of combination therapy of antihistamines with montelukast and corticosteroid nasal spray.

\section{Conclusions}

This is one of the first case reports showing improvement of TN symptoms with combination therapy of antihistamines, leukotriene receptor antagonists, and corticosteroids. More clinical studies and trials are needed to study the effectiveness of these drugs in the treatment of TN.

\section{Additional Information \\ Disclosures}

Human subjects: Consent was obtained by all participants in this study. Conflicts of interest: In compliance with the ICMJE uniform disclosure form, all authors declare the following: Payment/services info: All authors have declared that no financial support was received from any organization for the submitted work. Financial relationships: All authors have declared that they have no financial relationships at present or within the previous three years with any organizations that might have an interest in the submitted work. Other relationships: All authors have declared that there are no other relationships or activities that could appear to have influenced the submitted work.

\section{References}

1. Turton M, Malan-Roux P: Trigeminal neuralgia: case report and literature review. Stomatological Dis Sci. 2019, 3:7-10. 10.20517/2573-0002.2019.08

2. Saraswathi GK, Swathi T: Trigeminal neuralgia: a case report with review of literature . SAJ Case Rep. 2017, 4:102.

3. Obara I, Telezhkin V, Alrashdi I, Chazot PL: Histamine, histamine receptors, and neuropathic pain relief . Br J Pharmacol. 2020, 177:580-599. 10.1111/bph.14696

4. Headache classification committee of the International Headache Society (IHS) The international classification of headache disorders, 3rd edition. Cephalalgia. 2018, 38:1-211. 10.1177/0333102417738202

5. Maarbjerg S, Gozalov A, Olesen J, Bendtsen L: Concomitant persistent pain in classical trigeminal neuralgia: 


\section{Cureus}

evidence for different subtypes. Headache. 2014, 54:1173-1183. 10.1111/head.12384

6. Nurmikko TJ, Eldridge PR: Trigeminal neuralgia -- pathophysiology, diagnosis, and current treatment . Br J Anaesth. 2001, 87:117-132. 10.1093/bja/87.1.117

7. Katusic S, Beard CM, Bergstralth E, Kurland LT: Incidence and clinical features of trigeminal neuralgia, Rochester, Minnesota, 1945-1984. Ann Neurol. 1990, 27:89-95. 10.1002/ana.410270114

8. Cruccu G, Gronseth G, Alksne J, et al.: AAN-EFNS guidelines on trigeminal neuralgia management . Eur J Neurol. 2008, 15:1013-1028. 10.1111/j.1468-1331.2008.02185.x

9. Horton CE, Brennan AJ: Use of antihistamine drugs in the treatment for trigeminal neuralgia . JAMA. 1948, 27:870-872. 10.1001/JAMA.1948.72890300001006

10. Hanes WJ: Clinical research on the etiology and treatment of tic douloureux on an allergic basis: report of 183 cases. Oral Surg Oral Med Oral Pathol. 1967, 23:728-736. 10.1016/0030-4220(67)90361-1 